\title{
On the geometry of Banach spaces with modulus of convexity of power type 2
}

\author{
by \\ M. Ivanov (Sofia), A. J. Pallares (Murcia) and \\ S. Troyanski (Murcia)
}

\begin{abstract}
We use one-dimensional differential inequalities to estimate the squareness and type of Banach spaces with modulus of convexity of power type two. The estimates obtained are sharp and the constants involved moderate.
\end{abstract}

1. Introduction. The moduli of convexity and smoothness of a Banach space $X$,

$$
\delta_{X}(\varepsilon)=\inf \left\{1-\left\|\frac{x+y}{2}\right\|:\|x\|=\|y\|=1,\|x-y\|=\varepsilon\right\}, \quad 0 \leq \varepsilon \leq 2,
$$

and

$$
\rho_{X}(\tau)=\sup \left\{\frac{\|x+\tau y\|+\|x-\tau y\|-2}{2}:\|x\|=\|y\|=1\right\}, \quad \tau \geq 0,
$$

respectively, play an important role in Banach space theory. The duality between them reveals itself in the Lindenstrauss formula (e.g. [13, p. 61])

$$
\rho_{X^{*}}(\tau)=\sup \left\{\tau \varepsilon / 2-\delta_{X}(\varepsilon): 0 \leq \varepsilon \leq 2\right\} .
$$

According to the Nordlander Theorem [17], every Hilbert space $H$ is in a sense the most convex and the most smooth among Banach spaces, that is,

$$
\delta_{X}(\varepsilon) \leq \delta_{H}(\varepsilon)=1-\sqrt{1-\varepsilon^{2} / 4}=\varepsilon^{2} / 8+O\left(\varepsilon^{4}\right)
$$

and

$$
\rho_{X}(\varepsilon) \geq \rho_{H}(\varepsilon)=\sqrt{1+\tau^{2}}-1=\tau^{2} / 2+O\left(\tau^{4}\right)
$$

for arbitrary $X$. We write the Taylor expansion not only for the sake of greater clarity but also because it is the asymptotic behaviour at 0 of $\delta_{X}$ and $\rho_{X}$ which matters the most.

2010 Mathematics Subject Classification: 46B03, 46B20.

Key words and phrases: Banach space geometry, moduli of convexity and smoothness, Banach-Mazur distance. 
In this connection, it is rather difficult to compute precisely, or even up to equivalence, the modulus of convexity (or smoothness) of a given space. Notable among the special cases for which such calculations were carried through are $L_{p}$ spaces [8] (see also [15]), more general Orlicz spaces [14] (see also [6]), and Lorentz spaces [1, 2].

By a surprisingly simple argument Figiel [6] proved a sharp estimate for the case when $X$ is a $p$-convex and $q$-concave Banach lattice, $1<p \leq 2 \leq$ $q<\infty$ :

$$
\delta_{X}(\varepsilon) \geq \frac{p-1}{8} \varepsilon^{q} .
$$

(For the definition of $p$-convexity and $q$-concavity we refer to [13, p. 40].)

Recently the class of Banach spaces with modulus of convexity of power type 2 has been studied intensively in connection with the problem of extension of Lipschitz mappings [16, 3, 4].

In [9] we estimate some geometrical quantities for a Banach space with modulus of convexity of power type 2 in terms of the constant $p \in(1,2]$ (see (4) below). In this paper we elaborate on the method of [9] in order to find new asymptotically sharp estimates.

Before stating the main result we make the convention that $k, k_{1}, k_{2}, \ldots$ will denote positive absolute constants. In particular, we set

$$
\begin{aligned}
k & =2+\sqrt{2}, \\
k_{2} & =k / 2 .
\end{aligned}
$$

We also recall a couple of well-known notions. The quantity

$$
d_{2}(X)=\sup \left\{d\left(Y, l_{2}^{(2)}\right): Y \subset X, \operatorname{dim} Y=2\right\},
$$

where $d$ is the Banach-Mazur distance, obviously measures how far from an ellipse the two-dimensional sections of $S_{X}$ are. By the John Theorem [11, $d_{2}(X) \leq \sqrt{2}$, and by the Jordan-von Neumann Theorem [12], if $d_{2}(X)=1$ then $X$ is Hilbert. So, we are inclined to assume that the smaller $d_{2}(X)$ the nicer $X$ is. Indeed, by the James Theorem, $X$ is superreflexive if, for some equivalent norm, $d_{2}(X)<\sqrt{2}$ (see [10]).

We refer to [13, p. 72] for the definition of type.

TheOrem 1.1. Suppose

$$
\liminf _{\varepsilon \rightarrow 0} \frac{\delta_{X}(\varepsilon)}{\varepsilon^{2}} \geq \frac{p-1}{8}
$$

for some $p \in(1,2]$. Then

$$
d_{2}(X) \leq \frac{(p-1)+\sqrt{2} k_{2}(2-p)}{(p-1)+k_{2}(2-p)} .
$$


(In particular, (5) holds for every p-convex and 2-concave Banach lattice.) Also,

$$
\text { type } X \geq 1+\frac{p-1}{p-1+\sqrt{2} k(2-p)} .
$$

We immediately get the following

Corollary 1.2. If the Banach space $X$ has no type greater than $p \in$ $(1,2]$ then

$$
\liminf _{\varepsilon \rightarrow 0} \frac{\delta_{X}(\varepsilon)}{\varepsilon^{2}} \leq \frac{1}{8}\left(1-\frac{\sqrt{2}(2-p)}{k(p-1)+\sqrt{2}(2-p)}\right) .
$$

(In particular, (7) holds for any Banach space which contains uniformly $l_{p}^{(n)}$ for all $n \in \mathbb{N}$.)

In conclusion we present some clarifying remarks.

Note first that from (5) it follows, as it should, that $d_{2}(X)<\sqrt{2}$ for all $p \in(1,2]$.

REMARK 1.3. The estimate (5) is asymptotically sharp when $p \rightarrow 1$ or $p \rightarrow 2$.

Indeed, let $\mathcal{Y}_{p}$ be the class of all Banach spaces that satisfy (4) and let

$$
D_{p}=\sup \left\{d_{2}(X): X \in \mathcal{Y}_{p}\right\} .
$$

From (1) we know that $l_{p}^{(2)} \in D_{p}$. Also, as is well-known, $d_{2}\left(l_{p}^{(2)}\right)=2^{1 / p-1 / 2}$. Therefore, (5) implies

$$
2^{1 / p-1 / 2} \leq D_{p} \leq \frac{(p-1)+\sqrt{2} k_{2}(2-p)}{(p-1)+k_{2}(2-p)} .
$$

Considering the difference between the rightmost and leftmost sides of the above as a function of $p \in[1,2]$, we see that it is zero at $p=1$ and $p=2$ and has bounded second derivative on $[1,2]$. So, this difference is smaller than $k_{1}(p-1)(2-p)$, meaning that the estimate (5) of $D_{p}$ is asymptotically sharp.

REMARK 1.4. The estimates (6) and (7) are also asymptotically sharp for both $p \rightarrow 1$ and $p \rightarrow 2$.

We reason in similar fashion using $l_{p}$ as an example: $l_{p} \in \mathcal{Y}_{p}$, type $l_{p}=p$ and $l_{p}$ has no type strictly greater than $p$.

REMARK 1.5. An asymptotically sharp estimate of the form (6) can be deduced from the renorming result of [9], since an equivalent norm with modulus of smoothness of power type $p$ implies type $p$ (e.g. [13, p. 100]). However, the constant $\sqrt{2} k$ in $(6)$ is much smaller than what can be obtained 
from [9]. Also, the main result of the latter depends upon much deeper results.

REMARK 1.6. For $X=l_{p}$ the constant $(p-1) / 8$ in (1) is the best possible, since $\delta_{l_{p}}(\varepsilon)=(p-1) \varepsilon^{2} / 8+o\left(\varepsilon^{2}\right)$ (see [8, 15]). It is interesting that for $p=2$ the constant $1 / 8$ is best possible even for equivalent norms, due to the Nordlander Theorem. It is open if the same is true for $l_{p}$.

2. Differential inequality. The proof of Theorem 1.1 is based on the following

Proposition 2.1. Let $r=r(\theta)$ be a real $2 \pi$-periodic function with absolutely continuous first derivative such that

(i) $0 \leq r\left(r^{\prime \prime}+r\right) \leq 1+a$ a.e. for some $a \geq 0$,

(ii) $0 \leq r \leq 1$,

(iii) for every closed interval I of length $\pi / 2$ there is $\alpha \in I$ with $r(\alpha)=1$, Then

$$
r \geq \frac{1+k_{2} a}{1+\sqrt{2} k_{2} a} .
$$

We postpone the proof of the proposition to the next section. Now we deduce Theorem 1.1 from it.

In 9] we have introduced the shorthand notation

$$
a(X)=2 \limsup _{\tau \rightarrow 0} \frac{\rho_{X}(\tau)}{\tau^{2}}-1, \quad b(X)=\left(8 \liminf _{\varepsilon \rightarrow 0} \frac{\delta_{X}(\varepsilon)}{\varepsilon^{2}}\right)^{-1}-1 .
$$

That is,

$$
\liminf _{\varepsilon \rightarrow 0} \frac{\delta_{X}(\varepsilon)}{\varepsilon^{2}}=\frac{1}{8(1+b(X))}, \quad \limsup _{\tau \rightarrow 0} \frac{\rho_{X}(\tau)}{\tau^{2}}=\frac{a(X)+1}{2},
$$

$a(X), b(X) \geq 0$ and $a\left(X^{*}\right)=b(X)$.

The main point is the following proposition whose proof is an elaboration on some techniques in [9].

Proposition 2.2. Each Banach space $X$ satisfies

$$
d_{2}(X) \leq \frac{1+\sqrt{2} k_{2} b(X)}{1+k_{2} b(X)}
$$

and

$$
\log _{2} d_{2}(X) \leq \frac{k b(X)}{2(\sqrt{2}+k b(X))} .
$$

Proof. Since we are concerned with estimating, for the case $\operatorname{dim} X=2$, the quantity

$$
d_{2}(X):=\inf \left\{\|T\|\left\|T^{-1}\right\| ; T: X \leftrightarrow l_{2}^{2}\right\}
$$


with respect to $b(X)$, and $d_{2}(X)=d_{2}\left(X^{*}\right)$, we assume that we are given $a=a\left(X^{*}\right)=b(X) \in(0, \infty)$ and try to estimate $d_{2}(X)$.

As in [9], we assume that $Y=X^{*}$ is realised on the plane $\mathbb{R}^{2}$ in such a way that the Euclidean sphere

$$
S=\left\{\left(x_{1}, x_{2}\right): x_{1}^{2}+x_{2}^{2}=1\right\}
$$

is the John sphere for $B_{Y}$. That is, the Euclidean norm $|\cdot|$ satisfies $|\cdot| \geq\|\cdot\|$ and there is no ellipse of area greater than $\pi$ included in $B_{Y}$. It is well known (see e.g. [5, p. 68] or [7]) that $|\cdot| \leq \sqrt{2}\|\cdot\|$. Let $e_{1}, e_{2}$ be the unit vector basis in $\mathbb{R}^{2}$ and $r(\theta)=\left\|\cos \theta e_{1}+\sin \theta e_{2}\right\|$. Then

$$
S_{Y}=\left\{\frac{1}{r(\theta)}(\cos \theta, \sin \theta): \theta \in[-\pi, \pi]\right\} .
$$

Since $\|\cdot\| \leq|\cdot| \leq \sqrt{2}\|\cdot\|$, we have $1 / \sqrt{2} \leq r(\theta) \leq 1$ for all $\theta$. In [19, Lemma 1] it is shown that each arc of the Euclidean sphere $S$ with Euclidean length $\pi / 2$ has a point of contact $w \in S \cap S_{Y}$. Thus, $r$ satisfies (ii) and (iii) of Proposition 2.1.

In [9, Lemma 3.1] it is shown that $r$ has Lipschitz continuous first derivative. Also in [9, pp. 385-386] it is proved that $r$ satisfies the right hand inequality of Proposition 2.1(i) for $a=a(Y)$, while the left hand inequality follows from the convexity of the unit ball.

We see that $r$ satisfies all the hypotheses of Proposition 2.1 and that $d_{2}(Y)=\max \{1 / r(\theta)\}$. So from (8) we get (9):

$$
d_{2}(X)=d_{2}(Y) \leq \frac{1+\sqrt{2} k_{2} a}{1+k_{2} a}=\frac{1+\sqrt{2} k_{2} b(X)}{1+k_{2} b(X)} .
$$

To complete the proof we need the following elementary inequality that follows from the convexity of the function $t \log t$ :

$$
2 \log _{2} t \leq \frac{\sqrt{2}(t-1)}{(\sqrt{2}-1) t}, \quad t \in[1, \sqrt{2}] .
$$

Also, the function on the right hand side of this inequality is increasing. Thus we can put $t=d_{2}(X)$ in this inequality and replace $t$ with the estimate of $d_{2}(X)$ given in $(9)$ to get

$$
2 \log _{2} d_{2}(X) \leq \frac{\sqrt{2} k_{2} b(X)}{1+\sqrt{2} k_{2} b(X)},
$$

which implies 10 .

In the proof Theorem 1.1 we will also use the following

Proposition 2.3 ([18, 19]). Each Banach space has type

$$
\frac{2}{1+2 \log _{2} d_{2}(X)} \text {. }
$$


Proof of Theorem 1.1. From (4) we have

$$
b(X) \leq \frac{1}{p-1}-1=\frac{2-p}{p-1} .
$$

The straightforward substitution in $(9)$ gives $(5)$.

Also, from Proposition 2.2 we get

$2 \log _{2} d_{2}(X) \leq 1-\frac{\sqrt{2}}{\sqrt{2}+k b(X)}=1-\frac{1}{1+k_{3} b(X)} \leq 1-\frac{p-1}{p-1+k_{3}(2-p)}$,

where $k_{3}=k / \sqrt{2}=1+\sqrt{2}$. So,

$$
1+2 \log _{2} d_{2}(X) \leq \frac{(p-1)+2 k_{3}(2-p)}{(p-1)+k_{3}(2-p)}
$$

and

$$
\frac{2}{1+2 \log _{2} d_{2}(X)} \geq \frac{2(p-1)+2 k_{3}(2-p)}{(p-1)+2 k_{3}(2-p)}=1+\frac{p-1}{(p-1)+2 k_{3}(2-p)},
$$

which together with Proposition 2.3 implies (6).

Proof of Corollary 1.2. Since $X$ has no type greater than $p$, Proposition 2.3 implies

$$
p \geq \frac{2}{1+2 \log _{2} d_{2}(X)} .
$$

This and 10$)$ give

$$
\frac{2}{p}-1 \leq 2 \log _{2} d_{2}(X) \leq \frac{k b}{2(\sqrt{2}+k b)},
$$

where $b=b(X)$. That is,

$$
k b \geq \sqrt{2} \frac{2-p}{p-1}
$$

and

$$
\frac{1}{1+b} \leq \frac{k(p-1)}{k(p-1)+\sqrt{2}(2-p)}=1-\frac{\sqrt{2}(2-p)}{k(p-1)+\sqrt{2}(2-p)}
$$

3. The proof of Proposition 2.1. For $\alpha \in(0, \pi / 4]$ and $m, t \geq 1$ we denote by $P_{\alpha, m, t}$ the following problem:

$$
P_{\alpha, m, t}\left\{\begin{array}{l}
0 \leq f^{\prime \prime}(\theta)+f(\theta) \leq t m \quad \text { for almost all } \theta \in(0, \alpha), \\
f(0)=m^{-1}, \quad f(\alpha)=1, \\
f^{\prime}(0)=f^{\prime}(\alpha)=0 .
\end{array}\right.
$$

We say that the function $f$ is a solution to $P_{\alpha, m, t}$ if $f^{\prime}$ is absolutely continuous on $[0, \alpha]$ and $f$ and its derivatives satisfy the above conditions.

Let $r(\theta)$ be a function that satisfies the hypothesis of Proposition 2.1 Translating the independent variable if necessary, we can assume that $r(0)=$ 
$\min \{r(\theta): \theta \in[-\pi, \pi]\}$. By property (iii) in the proposition with $I=$ $[-\pi / 4, \pi / 4]$ we can also assume that there is $\alpha \in(0, \pi / 4]$ such that $r(\alpha)=$ $1=\max \{r(\theta): \theta \in[-\pi, \pi]\}$, using a change of sign when $\alpha$ is negative. Thus our function $r$ is a solution of the problem $P_{\alpha, 1 / r(0), 1+a}$.

We prove our proposition by studying the solutions of $P_{\alpha, m, t}$. One simple fact we will use is given in the following

Lemma 3.1. Let $\beta \in(0, \pi)$ and the function $f:[0, \beta] \rightarrow \mathbb{R}$ be such that $f^{\prime}$ is absolutely continuous and

$$
f^{\prime \prime}+f \geq 0 \quad \text { a.e. on }(0, \beta) \text { and } f(0)=f^{\prime}(0)=0 .
$$

Then $f \geq 0$ on $[0, \beta]$.

Proof. Assume that there is $\beta_{1} \in[0, \beta]$ such that $f\left(\beta_{1}\right)<0$.

For small enough $\eta>0$ the function $f_{1}=f+\eta \sin t$ satisfies

$$
f_{1}^{\prime \prime}+f_{1}=f^{\prime \prime}+f \geq 0 \quad \text { a.e. on }(0, \beta),
$$

$f_{1}\left(\beta_{1}\right)<0, f_{1}(0)=0$ and $f_{1}^{\prime}(0)=\eta>0$. Because of the latter, $f_{1}$ is positive for small enough $t$, and since it is continuous and becomes negative at $\beta_{1} \in(0, \pi)$, there is $\beta_{2} \in\left(0, \beta_{1}\right)$ such that

$$
f_{1}(0)=f_{1}\left(\beta_{2}\right)=0, \quad f_{1}>0 \quad \text { on }\left(0, \beta_{2}\right) .
$$

Let $k=\pi / \beta_{2}$ and $g=\sin k t$. Then

$$
k>1
$$

and

$$
g(0)=g\left(\beta_{2}\right)=0, \quad g>0, \quad g^{\prime \prime}=-k^{2} g \quad \text { on }\left(0, \beta_{2}\right) .
$$

Integrating twice by parts and using (13) and (15) we get

$$
\int_{0}^{\beta_{2}} f_{1}^{\prime \prime} g d t=-k^{2} \int_{0}^{\beta_{2}} f_{1} g d t .
$$

Together with 12)-15, this implies

$$
0 \leq \int_{0}^{\beta_{2}}\left(f_{1}^{\prime \prime}+f_{1}\right) g d t=\left(1-k^{2}\right) \int_{0}^{\beta_{2}} f_{1} g d t<0,
$$

a contradiction.

The key to our proof of Proposition 2.1 is

Lemma 3.2. For $t \geq 1$ let

$$
\psi_{\alpha}(t)=\sup \left\{m: \text { there exists a solution to } P_{\alpha, m, t}\right\} .
$$

Then

$$
\psi_{\alpha}(t) \leq 2 \frac{t+\sqrt{2}-2}{\sqrt{2}+\sqrt{2} t-2}
$$


Proof. We fix $t \geq 1$ and observe that $\psi_{\alpha}(t) \geq 1$, since $f \equiv 1$ is a solution to $P_{\alpha, 1, t}$. We then fix $m \geq 1$ such that there is a solution, say $f$, to $P_{\alpha, m, t}$. In order to prove (17) it is enough to show that

$$
m \leq 2 \frac{t+\sqrt{2}-2}{\sqrt{2}+\sqrt{2} t-2} .
$$

Let $f_{1}(\theta)=f(\alpha-\theta)-\cos \theta$ for $\theta \in[0, \alpha]$. Since $f$ is a solution to $P_{\alpha, m, t}$ we see that a.e. on $(0, \alpha)$,

$$
f_{1}^{\prime \prime}(\theta)+f_{1}(\theta)=f^{\prime \prime}(\alpha-\theta)+f(\alpha-\theta) \geq 0,
$$

and $f_{1}(0)=f(\alpha)-1=0$, and $f_{1}^{\prime}(0)=f^{\prime}(\alpha)=0$, so Lemma 3.1 implies that $f_{1} \geq 0$ on $[0, \alpha]$. In other words,

$$
f(\theta)=f(\alpha-(\alpha-\theta)) \geq \cos (\alpha-\theta), \quad \forall \theta \in[0, \alpha] .
$$

Applying the same reasoning to the function $f_{2}(\theta)=t m+\left(m^{-1}-t m\right) \cos \theta-$ $f(\theta)$, satisfying $f_{2}^{\prime \prime}+f_{2}=t m-f-f^{\prime \prime} \geq 0, f_{2}(0)=m^{-1}-f(0)=0$, and $f_{2}^{\prime}(0)=-f^{\prime}(0)=0$, we get $f_{2} \geq 0$ on $[0, \alpha]$. That is,

$$
f(\theta) \leq t m+\left(m^{-1}-t m\right) \cos \theta, \quad \forall \theta \in[0, \alpha] .
$$

Combining (19) with 20, we obtain

$$
t m+\left(m^{-1}-t m\right) \cos \theta \geq \cos (\alpha-\theta)
$$

for all $\theta \in[0, \alpha]$. Equivalently,

$$
t m \geq g(\theta), \quad \forall \theta \in[0, \alpha],
$$

where $g(\theta)=\left(t m-m^{-1}\right) \cos \theta+\cos (\alpha-\theta)$.

Since $m, t \geq 1$ we get $t m-m^{-1} \geq 0$. Hence $g(\theta)>0$ for $\theta \in[0, \alpha]$. Since $g^{\prime}(\theta)=\sin (\alpha-\theta)-\left(t m-m^{-1}\right) \sin \theta, g^{\prime}(0)=\sin \alpha>0$ and

$$
g^{\prime}(\alpha)=-\left(t m-m^{-1}\right) \sin \alpha \leq 0,
$$

there is $\theta_{1} \in[0, \alpha]$ such that $g^{\prime}\left(\theta_{1}\right)=0$. Then from (21) it follows that

$$
\begin{aligned}
(t m)^{2} \geq & g^{2}\left(\theta_{1}\right)=g^{2}\left(\theta_{1}\right)+g^{\prime 2}\left(\theta_{1}\right) \\
= & {\left[\left(t m-m^{-1}\right) \cos \theta_{1}+\cos \left(\alpha-\theta_{1}\right)\right]^{2} } \\
& \quad+\left[\sin \left(\alpha-\theta_{1}\right)-\left(t m-m^{-1}\right) \sin \theta_{1}\right]^{2} \\
= & \left(t m-m^{-1}\right)^{2}+1+2\left(t m-m^{-1}\right) \cos \alpha .
\end{aligned}
$$

The latter is equivalent to

$$
2\left(t m-m^{-1}\right) \cos \alpha \leq 2 t-m^{-2}-1 \leq 2\left(t-m^{-2}\right)=2 m^{-1}\left(t m-m^{-1}\right) .
$$

Thus

$$
m<\cos ^{-1} \alpha \leq \sqrt{2},
$$

and moreover

$$
2 t-2 t m \cos \alpha \geq m^{-2}+1-2 m^{-1} \cos \alpha
$$


so that

$$
\begin{aligned}
t & \geq \frac{m^{2}+(1-2 m \cos \alpha)}{2 m^{2}(1-m \cos \alpha)}=\frac{1}{m^{2}}+\frac{m^{2}-1}{2 m^{2}(1-m \cos \alpha)} \\
& \geq \frac{1}{m^{2}}+\frac{\left(m^{2}-1\right)}{m^{2}(2-m \sqrt{2})}=\frac{1-\sqrt{2} m+m^{2}}{m^{2}(2-\sqrt{2} m)} .
\end{aligned}
$$

Let $h(u):=\left(u^{2}-\sqrt{2} u+1\right) / u^{2}$. Then

$$
t \geq \frac{h(m)}{2-\sqrt{2} m} .
$$

On the other hand, we have

$$
\begin{aligned}
h(u) & =1-\frac{\sqrt{2}}{u}+\frac{1}{u^{2}}, \quad h^{\prime}(u)=\frac{\sqrt{2}}{u^{2}}-\frac{2}{u^{3}}, \\
h^{\prime \prime}(u) & =-\frac{2 \sqrt{2}}{u^{3}}+\frac{6}{u^{4}}=\frac{2 \sqrt{2}}{u^{4}}\left(\frac{3}{\sqrt{2}}-u\right) .
\end{aligned}
$$

Since obviously $3 / \sqrt{2}>\sqrt{2}$, we find that $h^{\prime \prime} \geq 0$ and $h$ is convex for $u \in$ $[1, \sqrt{2}]$. In particular, $h$ is greater than its tangent at $(1, h(1))$. Since $h(1)=$ $2-\sqrt{2}$ and $h^{\prime}(1)=-(2-\sqrt{2})$, we get

$$
h(u) \geq(2-\sqrt{2})-(2-\sqrt{2})(u-1)=(2-\sqrt{2})(2-u), \quad u \in[1, \sqrt{2}] .
$$

From this and (23) it follows that

$$
t \geq(2-\sqrt{2}) \frac{2-m}{2-\sqrt{2} m} .
$$

Multiplying by $2-\sqrt{2} m \geq 0$ (see 22$)$, we obtain

$$
\begin{aligned}
2 t-(\sqrt{2} t) m & \geq 2(2-\sqrt{2})+(\sqrt{2}-2) m, \\
2(t+\sqrt{2}-2) & \geq(\sqrt{2}+\sqrt{2} t-2) m .
\end{aligned}
$$

Since $t \geq 1$ and thus $\sqrt{2}+\sqrt{2} t-2>0$, the latter is equivalent to 18 .

Proof of Proposition 2.1. Recall that by the assumptions at the beginning of this section, $r$ is a solution to $P_{\alpha, 1 / r(0), 1+a}$ and $r(0)=\min \{r(\theta): \theta \in$ $[-\pi, \pi]\}$.

From Lemma 3.2 we get

$$
\begin{aligned}
\frac{1}{r(0)} & \leq 2 \frac{1+a+\sqrt{2}-2}{\sqrt{2}+\sqrt{2}(1+a)-2}=\sqrt{2} \frac{a+\sqrt{2}-1}{a+2-\sqrt{2}} \\
& =\frac{\sqrt{2} a+2-\sqrt{2}}{a+2-\sqrt{2}}=\frac{1+\sqrt{2} k_{2} a}{1+k_{2} a}
\end{aligned}
$$

recalling that $k_{2}=1 /(2-\sqrt{2})=k / 2$. Hence we have (8):

$$
r \geq r(0) \geq \frac{1+k_{2} a}{1+\sqrt{2} k_{2} a} .
$$


Acknowledgments. M. Ivanov was partially supported by Bulgarian National Fund for Scientific Research contract DO 02-360/2008; by MM1401; and by Grant 105/2008 NIS, Sofia University.

A. J. Pallares was partially supported by Fundatión Séneca 08848/PI/08 CARM.

S. Troyanski was partially supported by MTM 2008-05396/MTM/Fondos FEDER and Fundatión Séneca 08048/PI/08 CARM; by Institute of Mathematics and Informatics, Bulgarian Academy of Sciences; and by Bulgarian National Fund for Scientific Research contract DO 02-360/2008.

\section{References}

[1] Z. Altshuler, Uniform convexity in Lorentz sequence spaces, Israel J. Math. 20 (1975), 260-274.

[2] - , The modulus of convexity of Lorentz and Orlicz sequence spaces, in: Notes in Banach Spaces, Univ. of Texas Press, Austin, TX, 1980, 359-378.

[3] K. Ball, Markov chains, Riesz transforms and Lipschitz maps, Geom. Funct. Anal. 2 (1992), 137-172.

[4] K. Ball, E. Carlen and E. Lieb, Sharp uniform convexity and smoothness inequalities for trace norms, Invent. Math. 115 (1994), 463-482.

[5] B. Bollobás, Linear Analysis, Cambridge Univ. Press, Cambridge, 1999.

[6] T. Figiel, On the moduli of convexity and smoothness, Studia Math. 56 (1976), $121-155$.

[7] A. A. Giannopoulos and V. D. Milman, Euclidean structure in finite dimensional normed spaces, in: Handbook of the Geometry of Banach Spaces, W. B. Johnson and J. Lindenstrauss (eds.), Elsevier, Amsterdam, 2001, 707-779.

[8] O. Hanner, On the uniform convexity of $L^{p}$ and $l^{p}$, Ark. Mat. 3 (1956), 239-244.

[9] M. Ivanov and S. Troyanski, Uniformly smooth renorming of Banach spaces with modulus of convexity of power type 2, J. Funct. Anal. 237 (2006), 373-390.

[10] R. C. James, Uniformly non-square Banach spaces, Ann. of Math. 80 (1964), 542550 .

[11] F. John, Extremum problems with inequalities as subsidiary conditions, in: Studies and Essays Presented to R. Courant, Interscience, 1948, 187-204.

[12] P. Jordan and J. von Neumann, On inner products in linear, metric spaces, Ann. of Math. 36 (1935), 719-723.

[13] J. Lindenstrauss and L. Tzafriri, Classical Banach Spaces. II: Function Spaces, Springer, 1979.

[14] R. Maleev and S. Troyanski, On the moduli of convexity and smoothness in Orlicz spaces, Studia Math. 54 (1975), 131-141.

[15] A. Meir, On the uniform convexity of $L^{p}$ spaces, $1<p \leq 2$, Illinois J. Math. 28 (1984), 420-424.

[16] A. Naor, Y. Peres, O. Schramm and S. Sheffield, Markov chains in smooth Banach spaces and Gromov-hyperbolic metric spaces, Duke Math. J. 134 (2006), 165-197.

[17] G. Nordlander, The modulus of convexity in normed linear spaces, Ark. Mat. 4 (1960), 15-17.

[18] G. Pisier, Sur les espaces qui ne contiennent pas de $l_{n}^{1}$ uniformément, in: Sém. Maurey-Schwartz 1973-1974, exp. 7, Centre de Math., École Polytech., Paris, 1974. 
[19] S. A. Rakov, Uniformly smooth renormings of uniformly convex Banach spaces, J. Soviet Math. 31 (1985), 2713-2721.

M. Ivanov

Faculty of Mathematics and Informatics

Sofia University

5, J. Bourchier blvd.

1164 Sofia, Bulgaria

E-mail: milen@fmi.uni-sofia.bg
A. J. Pallares, S. Troyanski Departamento de Matemáticas Universidad de Murcia Campus de Espinardo 30100 Murcia, Spain E-mail: apall@um.es stroya@um.es

Received May 21, 2009

Revised version September 7, 2009 